\title{
Melanoma of the Vagina: Case Report and Systematic Review of the Literature
}

\author{
VIOLETTA RAPI ${ }^{1}$, ASKIN DOGAN ${ }^{1}$, BEATE SCHULTHEIS ${ }^{2}$, FRANZISKA HARTMANN ${ }^{3}$, \\ GÜNTHER A. REZNICZEK ${ }^{1}$ and CLEMENS B. TEMPFER ${ }^{1}$ \\ Departments of ${ }^{1}$ Obstetrics and Gynecology, ${ }^{2}$ Medical Oncology and Hematology, \\ and ${ }^{3}$ Pathology, Ruhr University Bochum, Bochum, Germany
}

\begin{abstract}
Background: Primary melanoma of the vagina $(P M V)$ is a rare entity. The prognosis of women with PMV is poor and there is no standardized therapy for this type of malignancy. We present the case of a 72-year-old woman with PMV (cT2, pNO, MO). Case Report: Imaging studies showed no evidence of regional or distant metastases. Molecular analysis demonstrated wild-type B-Raf protooncogene, serine/threonine kinase (BRAF). Staging laparoscopy with pelvic lymphadenectomy and subsequent radiotherapy with 60 Gy delivered as pelvic teletherapy and vaginal brachytherapy was applied. Systematic literature review: A total of 805 cases of PMV were identified. Most lesions were melanotic (65\%) and localized (66\%), whereas amelanotic (35\%) and primary advanced lesions (34\%) were only seen in a minority of patients. BRAF mutation was detected in none out of 33 cases, tumor protein 53 (TP53) mutations and mast/stem cell growth factor receptor CD117 (KIT) amplification were identified in one case each. The most common treatment was surgery, reported in $43 \%$ of cases. Surgery combined with adjuvant radiotherapy, adjuvant immunotherapy (mostly with interferon-alpha), or adjuvant chemotherapy were given in 35\%, 8\%, and 3\% of cases, respectively. Radiotherapy or chemotherapy as sole treatments were used in 5\% and $1 \%$ of patients, respectively. Among patients with recurrence, chemotherapy (mostly dacarbazine) alone or in combination with surgery, radiotherapy or immunotherapy was the most common treatment in $61 \%$ of cases. The mean durations of
\end{abstract}

This Article is freely accessible online.

Correspondence to: Clemens Tempfer, MD, MBA, Department of Obstetrics and Gynecology, Ruhr-Universität Bochum - Marien Hospital Herne, Hölkeskampring 40, 44625 Herne, Germany. Tel: +49 23234991801, Fax: +49 23234993393, e-mail: clemens.tempfer@rub.de

Key Words: Melanoma, vagina, vaginal malignancy, immunotherapy, vaginectomy, brachytherapy, immune checkpoint inhibitors. recurrence-free and overall survival were 16.4 and 22.2 months, respectively. Conclusion: PMV is a rare malignancy with a poor prognosis. Surgery, radiotherapy, and immunotherapy with interferon-alpha are the mainstay of treatment for localized disease, while chemotherapy with dacarbazine is mostly used for unresectable and recurrent disease. No data on the clinical value of immune checkpoint inhibitors in PMV have been published.

Melanoma is caused by the malignant transformation of pigment cells. Apart from the skin, pigment cells such as melanocytes are present in various mucosal linings such as those of the gastrointestinal, respiratory, and urogenital tracts (1). Thus, in addition to the common cutaneous locations, melanomas may also arise in mucosal areas of various organs. Cutaneous melanomas are estimated to be the sixth most common cancer entity among females in the US (2). In contrast, mucosal melanomas are rare and account for only $1.4 \%$ of all melanomas. Of note, gender plays an important role in the epidemiology of mucosal melanomas. With almost three cases per million women, which is half that for men, mucosal melanomas are significantly more often diagnosed among women (3). This gender gap is caused by the fact that genital tract mucosal melanomas are more common among women than they are among men, whereas extra-genital mucosal melanomas are evenly distributed between the sexes (3).

In the female genital tract, the predominant location of melanoma is the vulva whereas the vagina is rarely affected (3). Specifically, the incidence of primary melanoma of the vagina (PMV) is about three cases per 10,000,000 women per year. Primary melanomas of the vulva are four to nine times more frequent than PMV $(4,5)$. PMV is typically diagnosed in elderly women at an advanced stage and is characterized by early recurrence and a poor prognosis (2). The 5-year overall survival rate for PMV is below $20 \%$ compared to around $45 \%$ for those with vulvar melanoma and $>80 \%$ for those with cutaneous melanoma (2-6). This remarkable difference has been attributed to a variety of 
factors, among them poorer visibility of PMV compared to cutaneous melanomas, late diagnosis, anatomical proximity to the vulvovaginal venous plexus, and tumor biology. Moreover, the relatively high rate of amelanotic tumors among PMV may also result in a later diagnosis.

In addition to the clinical behavior, the molecular characteristics of PMV are also markedly different from those of cutaneous melanomas and vulvar melanomas. For example, cutaneous melanomas have mutations in B-Raf proto-oncogene, serine/threonine kinase $(B R A F)$ in up to $66 \%$ of cases, whereas they are virtually absent in PMV (7, 8). In addition, vulvar and vaginal melanomas significantly differ in their molecular characteristics regarding mast/stem cell growth factor receptor CD117 (KIT) mutations. Aulmann et al. investigated 65 cases of PMV and vulvar melanomas for mutations in $B R A F$, N-rat sarcoma virus proto-oncogene $(N R A S)$, and KIT genes, and KIT gene amplification (8) They found that none of the PMV cases had BRAF mutations, whereas NRAS mutations and KIT amplifications were detected in $12 \%$ of both vulvar and vaginal tumors. In contrast, KIT mutations were present in $18 \%$ of vulvar melanomas but in none of the cases of PMV. Moderate or strong KIT protein expression was detected in all tumors with KIT mutations and most of the tumors with KIT amplification. In accordance with these results, Rouzbahman et al. found $B R A F$ mutations in $8 \%, K I T$ mutations in $28 \%, N R A S$ mutations in $28 \%$, and TP53 mutations in $8 \%$ of vulvar melanomas, whereas they detected only one TP53 mutation in the investigated PMV (9). Taken together, these findings suggest that in spite of their close anatomic proximity, the ontogenetic developments of vulvar and vaginal melanomas follow different molecular pathways.

To date, there is no consensus regarding the optimal management of women with PMV $(1,3,10)$. Specifically, it is unclear if treatment strategies should follow current practice regarding female genital tract cancer or should be extrapolated from established strategies used for cutaneous melanomas (11, 12). In addition, the clinical rationale of molecular tumor characterization of PMV regarding $B R A F, K I T$, or $N R A S$ as well as the therapeutic value of immunotherapies and targeted therapies such as the monoclonal antibodies to the programmed cell death protein-1 (PD-1) nivolumab and pembrolizumab and the antibody to the cytotoxic Tlymphocyte-associated protein 4 (CTLA-4) ipilimumab has not been established for patients with PMV (13).

To highlight the clinical characteristics, management, and prognosis of women with PMV, we report the case of a woman with histologically verified PMV and present a systematic review of the literature with cohort studies, case series, and case reports published about women with PMV. We discuss the most common therapies and respective outcomes.

\section{Case Report}

A 72-year-old woman was admitted to our outpatient dysplasia clinic in July 2017 with a vaginal mass of unclear nature for further evaluation. She reported no local pain or vaginal bleeding. She had a history of synchronous bilateral breast cancer diagnosed in 2015, treated with bilateral breastconserving surgery, breast irradiation, and endocrine therapy with an aromatase inhibitor for 5 years. Vaginal examination revealed a solid, black and grey solid mass of $66 \mathrm{~cm}$ in the lower third of the anterior vaginal wall extending to the right and left vaginal wall. The upper two-thirds of the vagina and the vaginal fornices were free of disease. Both parametria and the rectum were free on palpation. There was no inguinal lymphadenopathy. The external genitalia, uterus, and ovaries also appeared normal. A biopsy of the mass was performed and demonstrated PMV with polymorphic tumor cells, brown cytoplasm, tumor necrosis, and a high mitotic figure rate ( 8 mitoses per 10 high-power fields). Figure 1 shows hematoxylineosin and immunohistochemical stains of the PMV demonstrating expression of S100 and melan-A, as well as a low expression of pan-cytokeratin MNF116. Molecular analysis showed negativity for p.G469A (c.1406T>A), p.D594G (c.1781A $>$ G), and p.V600E (c.1799T >A) mutations of the $B R A F$ gene. Thus, the tumor was classified as wild-type $B R A F$.

Staging procedures, including computed tomography of the chest, mammography, and magnetic resonance tomography of the pelvis and abdomen, showed no signs of regional or distant metastases. Liver cirrhosis consistent with a history of alcohol abuse was diagnosed. Total body skin examination showed no evidence of cutaneous melanoma. The patient was treated with laparoscopic pelvic lymph node staging and subsequent vaginal brachytherapy with $50 \mathrm{~Gy}$. Adjuvant immunotherapy with interferon-alpha was refused by the patient. At the time of this report, the patient was alive and with no evidence of disease.

\section{Literature Review}

In a systematic literature search of the PubMed and Cochrane Central Register of Controlled Trials databases (search date 15-06-2017; time restriction to the last 20 years 1997-2017) using the search terms ("melanoma"[MeSH Terms] OR "melanoma"[All Fields]) AND ("vagina"[MeSH Terms] OR "vagina"[All Fields]), we identified 67 citations reporting on women with PMV, defined for the purpose of this review as primary melanocytic or amelanocytic melanoma of the vagina with or without regional/distant metastases, but without concurrent cutaneous manifestation of melanoma $(6,8,9,11,14-76)$. Studies not reporting on women with PMV, double publications, and studies reporting on women with vulvar melanomas or melanomas metastatic to the vagina were excluded. The 67 identified studies were 

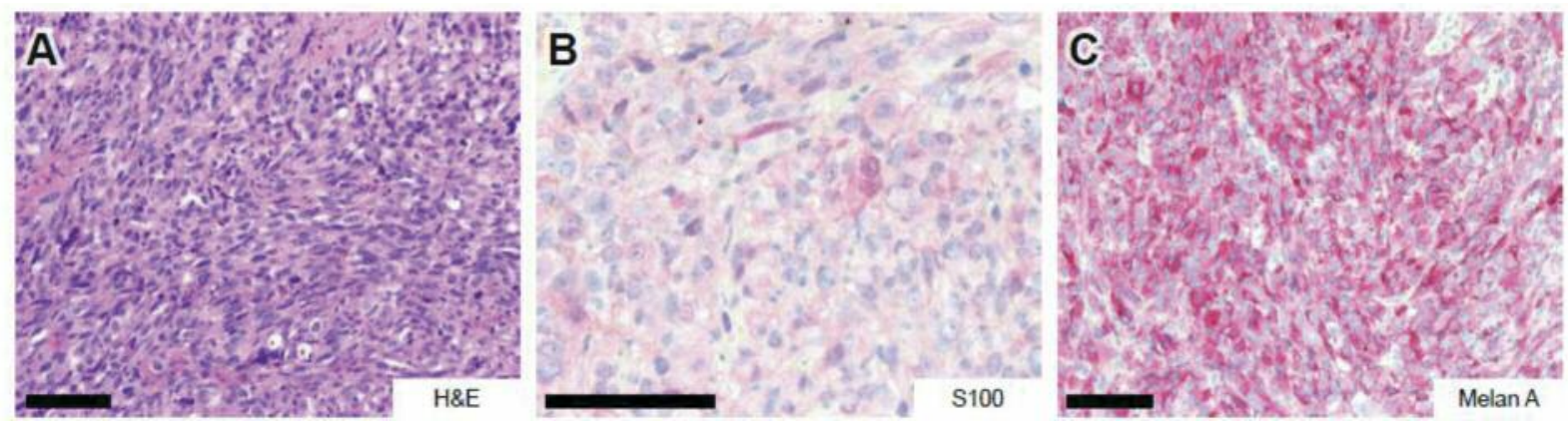

Figure 1. Hematoxylin-eosin $(H \& E)(A)$ and immunohistochemical (B to C) stains for $S 100(B)$ and melan-A $(C)$ of a primary melanoma of the vagina. The specimens demonstrate positive expression of S100 and melan-A. Bars, $50 \mu \mathrm{m}$ and $20 \mu \mathrm{m}$.

retrieved in full and were analyzed for this review. Figure 2 shows a flow diagram of the literature search algorithm.

Among the 67 studies, we found three retrospective cohort studies, two studies with data from national cancer registries, 20 retrospective cases series, and 42 case reports. No prospectively collected data were identified. Sixteen studies reported on $>10$ patients with PMV describing 11 (22), 13 (44), $14(27,72), 15(8,35), 22(24,76), 23(50), 25(68), 26(61)$, 31 (30), 37 (38), and 44 (28) cases, respectively. The two studies with data from national cancer registries described 192 (71) and 201 (31) cases of PMV. Table I shows the study characteristics and outcomes of patients with PMV described in all 67 studies. In summary, 805 cases of PMV have been reported in the literature during the past 20 years. At the time of first presentation, singular and multiple lesions were described in 102 (96\%) and four (4\%) cases, respectively. Most lesions were melanotic $(65 \%)$ and localized $(66 \%)$, whereas amelanotic $(35 \%)$ and primary advanced lesions $(34 \%)$ were only seen in a minority of patients. Only four studies with a total of 33 cases assessed the molecular characteristics of PMV. In these studies, $B R A F$ mutation was not identified in any of the investigated PMVs (0/33). TP53 mutations and KIT amplification were identified in one case each.

Treatment modalities and outcomes are shown in Table II. In a pooled analysis, the treatment most commonly used for PMV was surgery without any further treatment modality, which was reported in $43 \%$ of cases. Surgery combined with adjuvant radiotherapy, adjuvant immunotherapy (mostly interferonalpha), or adjuvant chemotherapy (mostly dacarbazine) were given in $35 \%, 8 \%$, and $3 \%$ of cases, respectively. Radiotherapy or chemotherapy as exclusive primary treatment were used in $5 \%$ and $1 \%$ of patients, respectively.

Among patients with recurrence of PMV, in contrast, chemotherapy alone or in combination with surgery, radiotherapy or immunotherapy was the most commonly used treatment, with $61 \%$ of cases. There is no standard chemotherapy regimen for PMV, but dacarbazine was the

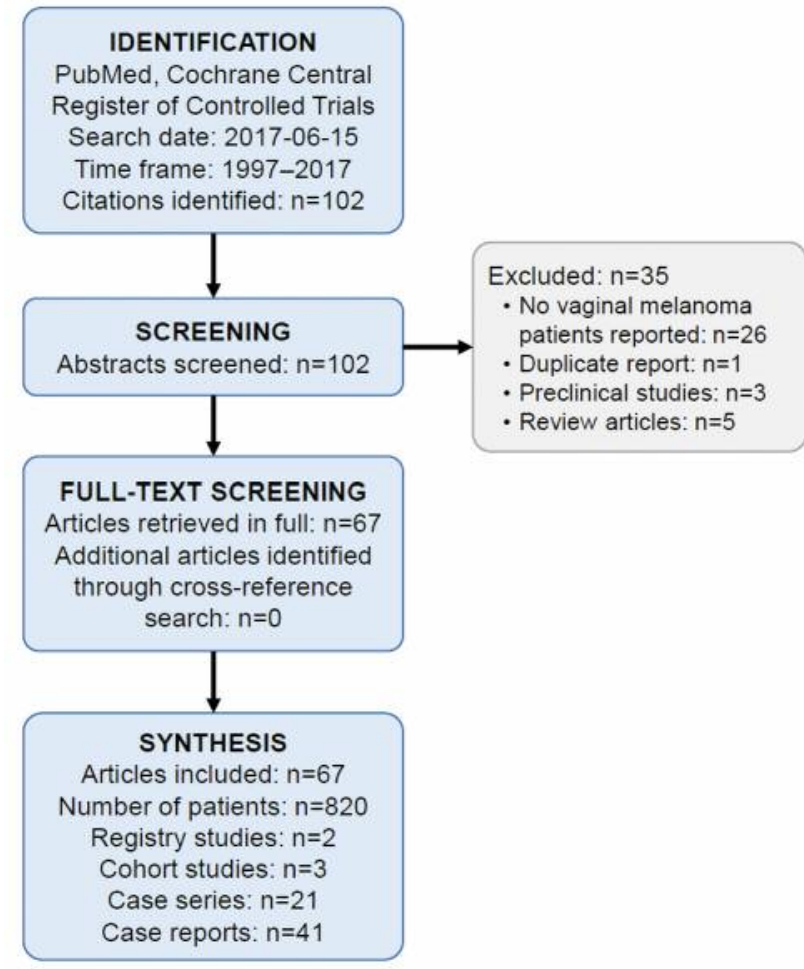

Figure 2. Flow diagram of the literature search algorithm. PMV, Primary melanoma of the vagina.

most commonly used substance; it was administered alone or in combination with vincristine, cisplatin/carboplatin, or nimustine. Overall, the prognosis of women with PMV was poor. The mean duration of recurrence-free survival was short at 16.4 months and the mean overall survival of women with PMV was only 22.2 months.

Immunotherapy was reported in a minority of patients both in primary and recurrent settings. Specifically, interferon-alpha was reported in 43/613 (7\%) cases and in 
Table I. Clinical studies describing women with primary melanoma of the vagina.

\begin{tabular}{|c|c|c|c|c|c|c|c|}
\hline Author & Year & $\begin{array}{l}\text { Study } \\
\text { type }\end{array}$ & $\begin{array}{l}\text { Number } \\
\text { of cases } \\
(n)\end{array}$ & $\begin{array}{l}\text { Singular/ } \\
\text { multiple } \\
\text { lesions }(\mathrm{n} / \mathrm{n})\end{array}$ & $\begin{array}{c}\text { Melanotic/ } \\
\text { amelanotic/mixed } \\
(\mathrm{n} / \mathrm{n} / \mathrm{n})\end{array}$ & $\begin{array}{c}\text { Localized/ } \\
\text { primary advanced } \\
(\mathrm{n} / \mathrm{n})\end{array}$ & $\begin{array}{l}\text { Molecular } \\
\text { profile }\end{array}$ \\
\hline Tasaka et al. (6) & 2017 & $\mathrm{CS}$ & 5 & & $3 / 2$ & $5 / 0$ & - \\
\hline Skovsted et al. (14) & 2017 & CS & 8 & - & - & - & - \\
\hline Kalampokas et al.(15) & 2017 & $\mathrm{CR}$ & 1 & $1 / 0$ & $1 / 0$ & $1 / 0$ & $B R A F \mathrm{wt}, K I T \mathrm{wt}$ \\
\hline Agarwal et al. (16) & 2017 & $\mathrm{CR}$ & 1 & $1 / 0$ & $1 / 0$ & $1 / 0$ & - \\
\hline Lee et al. (17) & 2016 & $\mathrm{CR}$ & 1 & $1 / 0$ & $0 / 1$ & $1 / 0$ & - \\
\hline Pank et al. (18) & 2016 & CR & 1 & - & - & - & - \\
\hline Rema et al. (19) & 2016 & CR & 1 & - & - & $0 / 1$ & - \\
\hline Todo et al. (20) & 2016 & CS & 10 & - & - & $1 / 9$ & - \\
\hline Stefanovic et al. (21) & 2015 & $\mathrm{CR}$ & 1 & $1 / 0$ & - & $1 / 0$ & - \\
\hline Rouzbahman et al. (9) & 2015 & $\mathrm{CS}$ & 11 & - & - & - & TP53 mut $(\mathrm{n}=1)$ \\
\hline Ishida et al. (22) & 2015 & $\mathrm{CR}$ & 1 & $1 / 0$ & $0 / 1$ & $0 / 1$ & - \\
\hline Liu et al. (23) & 2015 & $\mathrm{CS}$ & 4 & - & - & - & - \\
\hline Seifried et al. (24) & 2015 & $\cos$ & 22 & - & - & - & - \\
\hline Tsvetkov et al. (25) & 2014 & $\mathrm{CR}$ & 1 & $0 / 1$ & - & $0 / 1$ & - \\
\hline Chen et al. (26) & 2014 & $\mathrm{CR}$ & 1 & $1 / 0$ & $0 / 1$ & $1 / 0$ & - \\
\hline Aulmann et al. (8) & 2014 & $\mathrm{CS}$ & 15 & - & $4 / 11$ & - & $N R A S$ mut $(\mathrm{n}=2), K I T$ ampl $(\mathrm{n}=2)$ \\
\hline Karasawa et al. (27) & 2014 & $\cos$ & 14 & - & - & $0 / 14$ & - \\
\hline Xia et al. (28) & 2014 & CS & 44 & - & - & - & - \\
\hline Chaudhuri et al. (29) & 2013 & $\mathrm{CR}$ & 1 & $1 / 0$ & $1 / 0$ & $1 / 0$ & - \\
\hline Huang et al. (30) & 2013 & $\mathrm{CS}$ & 31 & - & $31 / 0$ & $29 / 2$ & - \\
\hline Kirschner et al. (31) & 2013 & $\cos$ & 201 & - & - & $129 / 84$ & - \\
\hline Kahr et al. (32) & 2013 & $\mathrm{CR}$ & 1 & $1 / 0$ & - & $1 / 0$ & - \\
\hline Chauhan et al. (33) & 2012 & $\mathrm{CR}$ & 1 & $1 / 0$ & $1 / 0$ & $1 / 0$ & - \\
\hline Gauthier et al. (34) & 2012 & CS & 6 & $6 / 0$ & - & $6 / 0$ & $K I T$ mut $(\mathrm{n}=1)$ \\
\hline Tcheung et al. (35) & 2012 & $\mathrm{CS}$ & 15 & - & - & - & - \\
\hline Lin et al. (36) & 2011 & $\mathrm{CR}$ & 1 & $1 / 0$ & $1 / 0$ & $1 / 0$ & - \\
\hline Albareda et al. (37) & 2011 & $\mathrm{CR}$ & 1 & $1 / 0$ & - & $1 / 0$ & - \\
\hline Frumovitz et al. (38) & 2010 & $\cos$ & 37 & $36 / 1$ & - & $37 / 0$ & - \\
\hline Greggi et al. (39) & 2010 & $\mathrm{CR}$ & 2 & $2 / 0$ & - & $1 / 1$ & - \\
\hline Samolis et al. (40) & 2010 & $\mathrm{CR}$ & 1 & $1 / 0$ & - & $1 / 0$ & - \\
\hline Das et al. (11) & 2010 & $\mathrm{CS}$ & 3 & $3 / 0$ & $1 / 2$ & $3 / 0$ & - \\
\hline Biswas et al. (41) & 2009 & CR & 1 & - & - & - & - \\
\hline Gungor et al. (42) & 2009 & $\mathrm{CS}$ & 4 & $3 / 1$ & - & $4 / 0$ & - \\
\hline Baloglu et al. (43) & 2009 & $\mathrm{CR}$ & 1 & $1 / 0$ & $1 / 0$ & $1 / 0$ & - \\
\hline Luo et al. (44) & 2008 & $\mathrm{CS}$ & 13 & - & - & - & - \\
\hline Takai et al. (45) & 2008 & $\mathrm{CR}$ & 1 & - & - & - & - \\
\hline Mukhopad-hyay et al. (46) & 2008 & $\mathrm{CR}$ & 1 & - & -- & - & - \\
\hline Grenader et al. (47) & 2008 & $\mathrm{CR}$ & 1 & $1 / 0$ & $0 / 1$ & $0 / 1$ & - \\
\hline Schmidt et al. (48) & 2008 & $\mathrm{CR}$ & 1 & $1 / 0$ & - & $0 / 1$ & - \\
\hline Ghosh et al. (49) & 2007 & CR & 1 & $1 / 0$ & $0 / 1$ & $1 / 0$ & - \\
\hline An et al. (50) & 2007 & $\mathrm{CS}$ & 23 & - & - & - & - \\
\hline Fulciniti et al. (51) & 2007 & $\mathrm{CR}$ & 1 & $1 / 0$ & $0 / 1$ & $1 / 0$ & - \\
\hline Ohno et al. (52) & 2007 & $\mathrm{CR}$ & 1 & $1 / 0$ & $1 / 0$ & $0 / 1$ & - \\
\hline Betschart et al. (53) & 2007 & $\mathrm{CR}$ & 1 & $1 / 0$ & $1 / 0$ & $0 / 1$ & - \\
\hline Androusopoul-os et al. (54) & 2005 & $\mathrm{CR}$ & 1 & $1 / 0$ & - & $1 / 0$ & - \\
\hline Gökaslan et al. (55) & 2005 & $\mathrm{CR}$ & 1 & $1 / 0$ & - & $1 / 0$ & - \\
\hline Oguri et al. (56) & 2004 & $\mathrm{CR}$ & 1 & $1 / 0$ & $0 / 1$ & $1 / 0$ & - \\
\hline Moros et al. (57) & 2004 & CR & 1 & $1 / 0$ & - & $1 / 0$ & - \\
\hline Siu et al. (58) & 2004 & CR & 1 & $1 / 0$ & $1 / 0$ & $1 / 0$ & - \\
\hline Mishra et al. (59) & 2003 & $\mathrm{CR}$ & 2 & - & $1 / 1$ & - & - \\
\hline Kim et al. (60) & 2003 & $\mathrm{CR}$ & 2 & $2 / 0$ & $2 / 0$ & $2 / 0$ & - \\
\hline Gupta et al. (61) & 2002 & $\mathrm{CS}$ & 26 & - & $20 / 6$ & $10 / 14^{*}$ & - \\
\hline Nakagawa et al. (62) & 2002 & $\mathrm{CR}$ & 1 & $0 / 1$ & $1 / 0$ & $1 / 0$ & - \\
\hline Piura et al. (63) & 2002 & $\mathrm{CR}$ & 1 & $1 / 0$ & - & $1 / 0$ & - \\
\hline Panek et al. (64) & 2001 & $\mathrm{CR}$ & 1 & $1 / 0$ & - & $1 / 0$ & - \\
\hline Fan et al. (65) & 2001 & $\mathrm{CR}$ & 1 & - & - & - & - \\
\hline
\end{tabular}


Table I. Continued

\begin{tabular}{|c|c|c|c|c|c|c|c|}
\hline Author & Year & $\begin{array}{l}\text { Study } \\
\text { type }\end{array}$ & $\begin{array}{l}\text { Number } \\
\text { of cases } \\
(n)\end{array}$ & $\begin{array}{c}\text { Singular/ } \\
\text { multiple } \\
\text { lesions }(\mathrm{n} / \mathrm{n})\end{array}$ & $\begin{array}{c}\text { Melanotic/ } \\
\text { amelanotic/mixed } \\
(\mathrm{n} / \mathrm{n} / \mathrm{n})\end{array}$ & $\begin{array}{c}\text { Localized/ } \\
\text { primary advanced } \\
(\mathrm{n} / \mathrm{n})\end{array}$ & $\begin{array}{l}\text { Molecular } \\
\text { profile }\end{array}$ \\
\hline Takehara et al. (66) & 2000 & $\mathrm{CR}$ & 1 & $1 / 0$ & $1 / 0$ & $1 / 0$ & - \\
\hline Li et al. (68) & 1999 & $\mathrm{CS}$ & 25 & - & - & - & - \\
\hline Rodier et al. (69) & 1999 & $\mathrm{CR}$ & 1 & $1 / 0$ & $1 / 0$ & $1 / 0$ & - \\
\hline Irvin et al. (70) & 1998 & $\mathrm{CS}$ & 7 & 6/- & - & $6 / 1$ & - \\
\hline Creasman et al. (71) & 1998 & COS & 192 & - & - & - & - \\
\hline Petru et al. (72) & 1998 & $\mathrm{CS}$ & 14 & $14 / 0$ & - & $9 / 5$ & - \\
\hline Stellato et al. (73) & 1998 & $\mathrm{CR}$ & 1 & $1 / 0$ & - & $1 / 0$ & - \\
\hline Wang et al. (74) & 1997 & $\mathrm{CS}$ & 9 & - & - & - & - \\
\hline Zarcone et al. (75) & 1997 & $\mathrm{CR}$ & 1 & $1 / 0$ & - & $1 / 0$ & - \\
\hline Liu et al. (76) & 1996 & $\mathrm{CS}$ & 22 & - & - & - & - \\
\hline Pooled Analysis & - & - & 805 & $\begin{aligned} 102(96 \%) / \\
\quad 4(4 \%)\end{aligned}$ & $\begin{array}{l}56(65 \%) / \\
29(35 \%)\end{array}$ & $\begin{array}{l}268(66 \%) / \\
137(34 \%)\end{array}$ & $\begin{array}{c}\text { BRAF mut }(0 / 33), \\
\text { TP53 mut }(1 / 33), \text { KIT ampl (1/33) }\end{array}$ \\
\hline
\end{tabular}

CS, Case series; CR, case report; wt, wild-type; COS, cohort study; mut, mutated; ampl, amplified; *data missing for $\mathrm{n}=2$.

2/18 (11\%) cases with detailed information on which therapeutic compound was used.

Data on targeted therapies or immune checkpoint inhibitors such as ipilimumab, nivolumab, or pembrolizumab were only reported in 2/805 cases of PMV. In these two cases, nivolumab was used alone and in combination with surgery and dacarbazine, both in the recurrent setting (6).

The largest cohort of women with PMV was published by two US cancer registries, the Surveillance, Epidemiology, and End Result (SEER) database (31) and the National Cancer Data Base (NCDB) (71). The study using SEER data comprised of 201 cases of PMV. In this study, the median age of affected women was 68 years. Sixty-four percent of the patient had early-stage PMV. Patients were operated upon in $70 \%$ of cases and had regional lymph node dissection in half of these patients and adjuvant radiotherapy in $40 \%$ of patients. Overall survival at 2 and 5 years were $24 \%$ and $15 \%$, respectively. The presence of metastases in regional lymph nodes was associated with a significantly worsened overall survival. Adjuvant radiation did not result in a statistically significant overall survival advantage compared to surgery alone (31).

The NCDB data comprised of 206 cases of PMV (71). Sixty-six percent of these patients were treated surgically and $40 \%$ of them received radiotherapy. Surgery as well as radiotherapy was used more frequently for patients with advanced-stage disease. The 5-year overall survival rate in this study was $14 \%$. The authors concluded that due to the limited evidence, the optimal therapy of PMV is unclear. However, it can be stated that younger patients were primarily treated with surgery, whereas primary radiotherapy was generally used in older patients and in those with advanced disease. Survival was poor and was primarily related to the level of tumor invasion. Specifically, the 3-year relative survival rate for patients with Clark levels of invasion II, II, IV, and V were $45 \%, 36 \%, 27 \%$, and $14 \%$, respectively.

Besides these two large registry studies, smaller cohort studies and case series describing between 11 (22) and 44 (28) patients with PMV were identified (8, 24, 27, 30, 35, 38, 44, $50,61,68,72,76)$. As expected, the heterogeneity among these studies with low numbers of patients with PMV was considerable. However, as shown in Table II, most patients were treated with surgery, whereas radiotherapy and chemotherapy were rarely used as primary therapy. For example, a large and well-documented cohort study, published by Frumovitz et al. (38) identified 37 patients with PMV treated between 1980 and 2009 (38). In this representative cohort of patients from the MD Anderson Cancer Center, vaginal bleeding was the most common presenting symptom. PMV were typically located in the distal third of the vagina (65\% of cases). Initial management included wide local or radical excision in $76 \%$ of patients, pelvic exenteration in $14 \%$, and radiotherapy, chemotherapy, or radiotherapy combined with chemotherapy in $10 \%$ of cases. Disease recurrence was observed in most patients 33/37 89 of cases Distant recurrence was observed in $88 \%$ of patients and was mostly located in the lungs and liver. Median progression-free survival was 11.4 months and median overall survival was 19 months. In contrast to other studies, radiotherapy after local wide excision reduced the risk of local recurrence and increased survival from 16.1 to 29.4 months.

The bulk of studies identified in our systematic review of the literature were case reports. The tumor characteristics and treatment modalities of these case reports are shown in Tables I and II. Among these 42 cases, long-term survivors were almost exclusively found in cases of localized disease at initial 
Table II. Treatment modalities and outcomes of women with primary melanoma of the vagina.

\begin{tabular}{|c|c|c|c|c|c|c|c|c|}
\hline Author & Year & $\begin{array}{l}\text { Treatment } \\
\text { modalities } \\
\text { (initial) }\end{array}$ & $\begin{array}{l}\text { Treatment } \\
\text { modalities } \\
\text { (recurrence) }\end{array}$ & $\begin{array}{c}\text { RM } \\
\text { (free/ } \\
\text { involved) }\end{array}$ & $\begin{array}{l}\mathrm{CHT} \\
\text { regimen } \\
\text { (n) }\end{array}$ & $\begin{array}{l}\text { RT (n)/ } \\
\text { Gy }\end{array}$ & $\begin{array}{c}\text { RFS } \\
\text { (months) }\end{array}$ & $\begin{array}{c}\text { OS } \\
\text { (months) }\end{array}$ \\
\hline Tasaka et al. (6) & 2017 & $\begin{array}{c}\mathrm{S}(\mathrm{n}=1), \mathrm{S}+\mathrm{IFN}(\mathrm{n}=3) \\
\text { S+ IFN+DAC+ } \\
\text { NIM+VIN }(\mathrm{n}=1)\end{array}$ & $\begin{array}{c}\mathrm{S}(\mathrm{n}=1), \mathrm{S}+\mathrm{DAC}+ \\
\mathrm{NIV}(\mathrm{n}=1), \mathrm{RS}(\mathrm{n}=1) \\
\mathrm{NIV}(\mathrm{n}=1)^{*}\end{array}$ & - & $\begin{array}{l}\text { DAC+NIM+ } \\
\text { VIN }(n=1), \\
\text { DAC }(n=1)\end{array}$ & - & $\begin{array}{c}13,3,8 \\
4,4\end{array}$ & $\begin{array}{c}26,14,10 \\
\quad 6,14\end{array}$ \\
\hline Skovsted et al. (14) & 2017 & - & - & - & - & - & - & - \\
\hline Kalampokas et al. (15) & 2017 & $S(n=1)$ & - & $1 / 0$ & - & - & 12 & 12 \\
\hline Agarwal et al. (16) & 2017 & DAC+VIN $(n=1)$ & - & - & DAC+VIN $(n=1)$ & - & - & - \\
\hline Lee et al. (17) & 2016 & $\mathrm{~S}+\mathrm{IFN}(\mathrm{n}=1)$ & $\begin{array}{c}\text { S+RT+DAC, CAR+ } \\
\text { DOC, CIS+VIN }(n=1)\end{array}$ & $1 / 0$ & $\begin{array}{c}\text { DAC }(\mathrm{n}=1), \text { CAR+ } \\
\text { DOC, CIS+VIN }(n=1)\end{array}$ & $1 / 48$ & 5 & 37 \\
\hline Rema et al. (19) & 2016 & $\mathrm{~S}+\mathrm{RT}(\mathrm{n}=1)$ & - & $1 / 0$ & - & $1 /-$ & 12 & 12 \\
\hline Todo et al. (20) & 2016 & $\begin{array}{c}\mathrm{S}(\mathrm{n}=1), \mathrm{S}+\mathrm{IFN}(\mathrm{n}=1) \\
\text { S+DAC+CIS+ } \\
\text { VIN }(\mathrm{n}=1), \mathrm{S}+\mathrm{DAC} \\
+\mathrm{NIM}+\mathrm{VIN}(\mathrm{n}=5) \\
\text { S+CAP }(\mathrm{n}=1)\end{array}$ & - & - & $\begin{array}{c}\text { DAC+CIS+ VIN }(n=1), \\
\text { DAC+NIM+VIN }(n=5), \\
\text { CAP }(n=1)\end{array}$ & 3 & $\begin{array}{c}56,46,6 \\
34,6,4,8 \\
\quad 6,5^{* *}\end{array}$ & $\begin{array}{c}65,46,12 \\
34,19,34 \\
10,18 \\
11,19^{* *}\end{array}$ \\
\hline Stefanovic et al. (21) & 2015 & $S(n=1)$ & - & $1 / 0$ & - & - & 5 & 5 \\
\hline Ishida et al. (22) & 2015 & $\begin{array}{l}\text { S+ DAC+NIM+ } \\
\text { VIN+IFN }(n=1)\end{array}$ & $S(n=1)$ & $1 / 0$ & $\begin{array}{c}\text { DAC+NIM+ } \\
\text { VIN }(n=1)\end{array}$ & - & 5 & 5 \\
\hline Tsvetkov et al. (25) & 2014 & $\mathrm{~S}+\mathrm{DAC}+\mathrm{BCG}(\mathrm{n}=1)$ & - & $1 / 0$ & $\operatorname{DAC}(n=1)$ & - & 21 & - \\
\hline Chen et al. (26) & 2014 & $\mathrm{~S}(\mathrm{n}=1)$ & - & $1 / 0$ & - & - & 3 & 8 \\
\hline Aulmann et al. (8) & 2014 & - & - & - & - & - & - & $\begin{array}{c}28,13,12 \\
117,23,14 \\
6,16,8 \\
19,28\end{array}$ \\
\hline Karasawa et al. (27) & 2014 & RT $(n=6)$ & - & - & - & $6 / 57.6$ & - & - \\
\hline Xia et al. (28) & 2014 & - & - & - & - & - & $14.4(n=44)$ & $39.5(n=44)$ \\
\hline Chaudhuri et al. (29) & 2013 & $S(n=1)$ & $\mathrm{RT}+\mathrm{TEM}(\mathrm{n}=1)$ & $1 / 0$ & $\operatorname{TEM}(n=1)$ & $1 / 40$ & 1 & 12 \\
\hline Huang et al. (30) & 2013 & $\begin{array}{c}\text { S }(n=7), S+I T \\
(n=15), \text { CHT+IT } \\
(n=4), \text { CHT }(n=3)\end{array}$ & - & - & - & - & - & - \\
\hline Kirschner et al. (31) & 2013 & $\begin{array}{c}\mathrm{S}(\mathrm{n}=69) \\
\mathrm{S}+\mathrm{RT}(\mathrm{n}=80)\end{array}$ & - & - & - & - & - & - \\
\hline Kahr et al. (32) & 2013 & $\mathrm{~S}(\mathrm{n}=1)$ & - & $1 / 0$ & - & - & 7 & - \\
\hline Chauhan et al. (33) & 2012 & $\mathrm{RT}(\mathrm{n}=1)$ & - & - & - & $1 /-$ & 12 & 12 \\
\hline Gauthier et al. (34) & 2012 & $S(n=4)$ & $\mathrm{S}(\mathrm{n}=3), \operatorname{CHT}(\mathrm{n}=2)$ & & & & $6,6,6$ & 12,83 \\
\hline Lin et al. (36) & 2011 & $\mathrm{~S}+\mathrm{IFN}(\mathrm{n}=1)$ & $\mathrm{S}+\mathrm{DAC}+\mathrm{IFN}(\mathrm{n}=1)$ & $1 / 0$ & $\operatorname{DAC}(n=1)$ & - & 7 & 30 \\
\hline Frumovitz et al. (38) & 2010 & $\begin{array}{c}\mathrm{S}(\mathrm{n}=12), \mathrm{S}+\mathrm{CHT} \\
(\mathrm{n}=1), \mathrm{S}+\mathrm{RT}(\mathrm{n}=20)\end{array}$ & - & $27 / 6$ & 20 & $\begin{array}{c}0 / 30(\mathrm{n}=10) \\
25(\mathrm{n}=2), \\
50(\mathrm{n}=8)\end{array}$ & ), - & - \\
\hline Greggi et al. (39) & 2010 & $S(n=2)$ & - & $2 / 0$ & - & - & - & - \\
\hline Samolis et al. (40) & 2010 & $\mathrm{~S}(\mathrm{n}=1)$ & - & $1 / 0$ & - & - & - & - \\
\hline Gungor et al. (42) & 2009 & $\begin{array}{l}\mathrm{S}(\mathrm{n}=1), \mathrm{S}+ \\
\operatorname{IFN}(\mathrm{n}=3)\end{array}$ & - & $4 / 0$ & - & - & $\begin{array}{r}24,3 \\
17,4\end{array}$ & $\begin{array}{c}24,5 \\
18,5\end{array}$ \\
\hline Baloglu et al. (43) & 2009 & $\mathrm{~S}+\mathrm{IFN}(\mathrm{n}=1)$ & $\mathrm{S}+\mathrm{CIS}+\mathrm{TEM}(\mathrm{n}=1)$ & $1 / 0$ & CIS+TEM $(n=1)$ & - & 9 & 18 \\
\hline Schmidt et al. (48) & 2008 & $S(n=1)$ & - & - & - & - & - & - \\
\hline Ghosh et al. (49) & 2007 & $\mathrm{~S}+\mathrm{RT}+\mathrm{DAC}(\mathrm{n}=1)$ & - & - & $\operatorname{DAC}(n=1)$ & $1 /-$ & 3 & 6 \\
\hline Fulciniti et al. (51) & 2007 & $\mathrm{~S}+\mathrm{IFN}(\mathrm{n}=1)$ & - & $1 / 0$ & - & - & - & - \\
\hline Ohno et al. (52) & 2007 & $\begin{array}{l}\text { RT+DAC+ } \\
\text { NIM+VIN }\end{array}$ & $\begin{array}{l}\mathrm{RT}+\mathrm{DAC}+\mathrm{NIM}+ \\
\mathrm{CIS}+\mathrm{TAM}(\mathrm{n}=1)\end{array}$ & - & $\begin{array}{r}\text { DAC+NIM+VIN }(\mathrm{n}=1) ; \\
\text { DAC+NIM+CIS }(\mathrm{n}=1)\end{array}$ & ; $1 / 57.6$ & 6 & 19 \\
\hline Betschart et al. (53) & 2007 & S+RT $(n=1)$ & DAC + THAL $(n=1)$ & $0 / 1$ & DAC $(n=1)$ & $1 / 59$ & 6 & - \\
\hline $\begin{array}{l}\text { Androusopoulos } \\
\text { et al. (54) }\end{array}$ & 2005 & $\mathrm{~S}+\mathrm{IFN}(\mathrm{n}=1)$ & - & - & - & - & - & 25 \\
\hline Gökaslan et al. (55) & 2005 & S+IFN $(n=1)$ & $\operatorname{DAC}(n=1)$ & $1 / 0$ & $\operatorname{DAC}(\mathrm{n}=1)$ & - & 9 & 16 \\
\hline Oguri et al. (56) & 2004 & $\mathrm{RT}+\mathrm{DAC}+\mathrm{CIS}+\mathrm{VIN}$ & - & - & DAC+CIS+ VIN $(n=1)$ & $1 / 64$ & 20 & 20 \\
\hline Moros et al. (57) & 2004 & $\mathrm{~S}+\mathrm{RT}+\mathrm{IFN}(\mathrm{n}=1)$ & - & $1 / 0$ & - & $1 / 56$ & 7 & 8 \\
\hline Siu et al. (58) & 2004 & $\mathrm{~S}+\mathrm{RT}(\mathrm{n}=1)$ & - & $1 / 0$ & - & $1 / 65$ & 2 & 2 \\
\hline
\end{tabular}


Table II. Continued

\begin{tabular}{|c|c|c|c|c|c|c|c|c|}
\hline Author & Year & $\begin{array}{l}\text { Treatment } \\
\text { modalities } \\
\text { (initial) }\end{array}$ & $\begin{array}{l}\text { Treatment } \\
\text { modalities } \\
\text { (recurrence) }\end{array}$ & $\begin{array}{c}\mathrm{RM} \\
\text { (free/ } \\
\text { involved) }\end{array}$ & $\begin{array}{l}\text { CHT } \\
\text { regimen } \\
\text { (n) }\end{array}$ & $\begin{array}{l}\mathrm{RT}(\mathrm{n}) / \\
\mathrm{Gy}\end{array}$ & $\begin{array}{c}\text { RFS } \\
\text { (months) }\end{array}$ & $\begin{array}{c}\text { OS } \\
\text { (months) }\end{array}$ \\
\hline Gupta et al. (61) & 2002 & $\begin{array}{c}\mathrm{S}(\mathrm{n}=4), \mathrm{RT}(\mathrm{n}=2) \\
\mathrm{S}+\mathrm{RT}(\mathrm{n}=7) ; \\
\mathrm{S}+\mathrm{CHT}(\mathrm{n}=1) \\
\mathrm{S}+\mathrm{RT}+\mathrm{CHT}(\mathrm{n}=11) \dagger\end{array}$ & - & $13 / 12^{\dagger}$ & - & - & - & $\begin{array}{c}37.7 \\
(n=23)\end{array}$ \\
\hline Nakagawa et al. (62) & 2002 & $\mathrm{~S}+\mathrm{RT}(\mathrm{n}=1)$ & - & $1 / 0$ & - & $1 / 24$ & - & - \\
\hline Piura et al. (63) & 2002 & $\mathrm{~S}+\mathrm{RT}(\mathrm{n}=1)$ & - & $1 / 0$ & - & $1 /-$ & 11 & 11 \\
\hline Panek et al. (64) & 2001 & $\mathrm{~S}+\mathrm{RT}(\mathrm{n}=1)$ & - & $1 / 0$ & - & $1 /-$ & 120 & 120 \\
\hline Takehara et al. (66) & 2000 & $\begin{array}{c}\text { DAC+CIS+ } \\
\text { NIM+TAM }(n=1)\end{array}$ & - & - & $\begin{array}{l}\text { DAC+CIS+ } \\
\text { NIM }(n=1)\end{array}$ & - & - & 13 \\
\hline Cobellis et al. (67) & 2000 & $S(n=15)$ & - & - & - & - & & $19(\mathrm{n}=15)$ \\
\hline Li et al. (68) & 1999 & $\begin{array}{c}\mathrm{S}(\mathrm{n}=17), \mathrm{CHT}+ \\
\text { IFN }(\mathrm{n}=8)\end{array}$ & - & - & - & - & - & $\begin{array}{r}19.5(\mathrm{n}=17) \\
8.3(\mathrm{n}=8)\end{array}$ \\
\hline Rodier et al. (69) & 1999 & $\mathrm{~S}+\mathrm{RT}(\mathrm{n}=1)$ & - & $1 / 0$ & - & $1 /-$ & 4 & 4 \\
\hline Irvin et al. (71) & 1998 & $\begin{array}{l}S(n=3), S+R T \\
(n=2), R T(n=2)\end{array}$ & - & - & - & $4 /-$ & $12.5(n=6)$ & $21.2(\mathrm{n}=7)$ \\
\hline Petru et al. (72) & 1998 & $\begin{array}{c}\mathrm{S}(\mathrm{n}=3), \mathrm{RT}(\mathrm{n}=5), \\
\mathrm{S}+\mathrm{RT}(\mathrm{n}=2), \mathrm{S}+\mathrm{DAC} \\
(\mathrm{n}=1), \mathrm{RT}+\mathrm{CAR} \\
(\mathrm{n}=1), \mathrm{RT}+\mathrm{DAC}+ \\
\text { VIN+BLEO }(\mathrm{n}=1)^{\dagger}\end{array}$ & - & - & $\begin{array}{c}\text { DAC+VIN+BLEO } \\
(\mathrm{n}=1), \text { DAC }(\mathrm{n}=1), \\
\text { CAR }(\mathrm{n}=1)\end{array}$ & 9/- & $\begin{array}{c}72,93,44 \\
28,7,23\end{array}$ & $30.6(n=14)$ \\
\hline Stellato et al. (73) & 1998 & $\mathrm{~S}(\mathrm{n}=1)$ & $\mathrm{DAC}+\mathrm{FOT}+\mathrm{IFN}(\mathrm{n}=1)$ & $1 / 0$ & DAC+FOT $(\mathrm{n}=1)$ & - & 4 & 12 \\
\hline Pooled Analysis & - & $\begin{array}{c}\text { S }(43 \%), \text { S+RT }(35 \%), \\
\text { S+IT }(8 \%), \text { RT }(5 \%), \\
\text { S+CHT (3\%), CHT+ } \\
\text { IT }(3 \%), \text { CHT }(1 \%), \\
\text { S+RT+IFN }(<1 \%), \\
\text { S+CHT+IT }(<1 \%)\end{array}$ & $\begin{array}{c}\text { CHT+/-S, IT, RT, } \\
\text { THAL (61\%), } \\
\text { S/RS }(33 \%), \\
\text { IT }(5 \%)\end{array}$ & $68 / 19$ & $\begin{array}{l}\text { DAC+/-VIN, CIS, } \\
=\text { NIM, CAR, } \\
\text { FOT }(23 / 28)\end{array}$ & - & Mean 16.4 & Mean 22.2 \\
\hline
\end{tabular}

RM, Resection margin; CHT, chemotherapy; RT, radiotherapy; RFS, recurrence-free survival; OS, overall survival; S, surgery; IFN, interferon- $\beta$; DAC, dacarbazine; NIM, nimustine; VIN, vincristine; RS, radiosurgery; CAR, carboplatin; DOC, docetaxel; CAP, cyclophosphamide, adriamycin, cisplatin; BCG, bacillus Calmette-Guerin vaccine; TEM, temozolamide; IT, immunotherapy including IFN, BCG, interleukin-2, measles vaccine, lymphokine-activated cells, and dendritic cells; CIS, cisplatin; TAM, tamoxifen; THAL, thalidomide; BLEO, bleomycin; FOT, fotemustine. *One patient refused treatment; **one patient lost to follow-up; ${ }^{\dagger}$ data missing for $\mathrm{n}=1 ;{ }^{\dagger}$ data missing for $\mathrm{n}=2$.

presentation and complete tumor resection with clear resection margins. For example, Panek et al. described the case of a woman with a small PMV, treated with surgical resection and vaginal brachytherapy, who survived for 10 years (64).

\section{Discussion}

In a case report and systematic review of the literature, we found that PMV is a rare female genital malignancy and carries a poor prognosis. Among 805 cases reported in the literature during the past 20 years, the mean duration of recurrence-free survival was short at 16 months and the mean overall survival time was only 22 months. No standard treatment for PMV has been established in the literature, but surgical excision either by local wide excision or radical surgery with colpectomy with/without exenteration is the mainstay of treatment for women with PMV. Many adjuvant treatment options have been described including radiotherapy, immunotherapy (mostly interferon-alpha), and chemotherapy (mostly dacarbazine). However, adjuvant radiotherapy and immunotherapy were only given in a minority of patients. Adjuvant chemotherapy was only used in $3 \%$ of cases. Among patients with recurrent PMV, chemotherapy alone or in combination with surgery, radiotherapy, or immunotherapy, on the other hand, was the most common treatment reported in $61 \%$ of cases. There is no standard chemotherapy regimen for PMV, but dacarbazine is the most commonly used substance. As for other types of melanomas, sensitivity to chemotherapy of PMV is low, thus underlining the need for molecularly based treatment strategies.

Despite the fact that targeted therapies such as the anti-PD1 monoclonal antibodies nivolumab and pembrolizumab and the anti-CTLA-4 antibody ipilimumab have been well established in patients with cutaneous melanomas, we 
identified such therapies were used in only two out of 805 cases of women with PMV. This is not surprising due to the rare occurrence of the disease and the fact that these therapies have only been available during the last couple of years. In the two cases described above, nivolumab was used alone and in combination with surgery and dacarbazine, both in the recurrent setting (6). In addition, the number of cases with molecular characterization was low. Based on these data, there is no evidence supporting the clinical efficacy of nivolumab, pembrolizumab, and ipilimumab in women with PMV. This is unfortunate because the clinical effectiveness of ipilimumab, nivolumab, and pembrolizumab has been clearly demonstrated in patients with primary advanced or recurrent metastatic cutaneous melanoma. Due to the rarity of PMV, clinical trials assessing the efficacy of these targeted treatments in PMV cannot be reasonably expected. Thus, efficacy assessments should be extrapolated from data on patients with cutaneous or mucosal melanoma, dependent on the molecular profile, or from anecdotal evidence for patients with PMV.

In accordance with the lack of data on the clinical efficacy of targeted therapies, molecular characterization of $B R A F$, $c K I T$, and NRAS as well as PD-1 surface expression were described in only four studies with 33 cases. In these studies, a $B R A F$ mutation was not identified in any of the investigated PMV (0/33). TP53 mutations and KIT amplification were only identified in one case each. Based on these data, there is no evidence for the clinical efficacy of nivolumab, pembrolizumab, and ipilimumab in women with PMV. In addition, BRAF, NRAS, KIT, and TP53 mutations were virtually absent among PMV cases. Thus, PMV might not be a suitable tumor entity for these targeted therapies. On the other hand, nivolumab has been demonstrated to be efficacious in cutaneous melanomas without $B R A F$ mutation (77). In a large, randomized phase III trial of 418 patients with previously untreated metastatic melanoma without BRAF mutation, nivolumab was superior to dacarbazine regarding tumor response (40\% vs. 14\%), progression-free survival (5.1 vs. 2.2 months), and overall survival (1-year survival: $73 \%$ vs. $42 \%$ ). Therefore, it is reasonable to believe that nivolumab may also be efficacious in PMV, which is typically $B R A F$-negative, as demonstrated above. We consider molecular characterization of PMV as important, firstly in order to identify possible therapeutic options, such as PD-1 inhibitors. Secondly, the $B R A F$ mutation status may also help to distinguish PMV from metastases of a cutaneous melanoma which might have already undergone apoptosis at the time of diagnosis.

Chemotherapy, although only rarely used in the adjuvant setting, was typically used in patients with recurrent disease. In our pooled analysis, chemotherapy was given to $61 \%$ of these patients. However, this high number of patients treated with chemotherapy has to be interpreted in light of the fact that targeted therapies were not available at the time these patients were treated. Chemotherapy was either administered as the sole treatment or in combination with surgery, radiotherapy or immunotherapy. The choice of drug regimen, clearly, was empirical and there is no standard chemotherapy regimen for PMV based on the available evidence. Among the many regimens and substances, dacarbazine was the most commonly used compound. In addition, vincristine, cisplatin/carboplatin, or nimustine have been used alone and in combination with dacarbazine. It is difficult to assess the benefit of chemotherapy in women with PMV. However, it can be clearly stated that PMV does not seem to be a very chemo-sensitive disease given that the mean overall survival of patients with PMV was only 22.2 months despite the use of chemotherapy in cases of recurrence.

Immunotherapy with interferon-alpha was used in $7 \%$ of patients as adjuvant treatment and in $11 \%$ of patients as treatment for recurrence. As with chemotherapy, it is difficult to assess the clinical benefit of interferon-alpha in patients with PMV due to lack of comparative studies.

In conclusion, we found that PMV is a rare form of melanoma with a poor prognosis. Surgery is the mainstay of treatment for localized disease while systemic chemotherapy and radiotherapy are appropriate for women with disseminated or recurrent PMV. Immunotherapy with interferon-alpha has been used in some patients and may be a useful adjunct therapy, but evidence for its efficacy is lacking.

\section{Declarations}

Ethics approval and consent to participate. Not applicable Consent for publication. Patient's consent for publication is available.

\section{Conflicts of Interest}

The Authors declare that they have no competing interests.

\section{References}

1 Mihajlovic M, Vlajkovic S, Jovanovic P and Stefanovic V: Primary mucosal melanomas: a comprehensive review. Int J Clin Exp Pathol 5(8): 739-753, 2012.

2 McLaughlin CC, Wu XC, Jemal A, Martin HJ, Roche LM and Chen VW: Incidence of noncutaneous melanomas in the U.S. Cancer 103: 1000-1007, 2005.

3 Leitao MM Jr., Cheng X, Hamilton AL, Siddiqui NA, Jurgenliemk-Schulz I, Mahner S, Åvall-Lundqvist E, Kim K and Freyer G: Gynecologic Cancer InterGroup (GCIG) consensus review for vulvovaginal melanomas. Int J Gynecol Cancer 24(9 Suppl 3): S117-122, 2014.

4 Weinstock M: Malignant melanoma of the vulva and vagina in the United States: Patterns of incidence and population-based estimates of survival. Am J Obstet Gynecol 171: 1225-1230, 1994.

5 Ragnarsson-Olding B, Johansson H, Rutqvist L and Ringborg U: Malignant melanoma of the vulva and vagina. Cancer 71: 18931897, 1993

6 Tasaka R, Fukuda T, Wada T, Kawanishi M, Imai K, Kasai M, Hashiguchi Y, Ichimura T, Yasui T and Sumi T: A retrospective 
clinical analysis of 5 cases of vaginal melanoma. Mol Clin Oncol 6(3): 373-376, 2017.

7 Lai X and Friedman A: Combination therapy for melanoma with BRAF/MEK inhibitor and immune checkpoint inhibitor: a mathematical model. BMC Syst Biol 11(1): 70, 2017.

8 Aulmann S, Sinn HP, Penzel R, Gilks CB, Schott S, Hassel JC, Schmidt D, Kommoss F, Schirmacher P and Kommoss S: Comparison of molecular abnormalities in vulvar and vaginal melanomas. Mod Pathol 27(10): 1386-1393, 2014.

9 Rouzbahman M, Kamel-Reid S, Al Habeeb A, Butler M, Dodge J, Laframboise S, Murphy J, Rasty G and Ghazarian D: Malignant melanoma of vulva and vagina: a histomorphological review and mutation analysis-a single-center study. J Low Genit Tract Dis 19(4): 350-353, 2015.

10 Bishop KD and Olszewski AJ: Epidemiology and survival outcomes of ocular and mucosal melanomas: A population-based analysis. Int J Cancer 134: 2961-2971, 2014.

11 Das P, Kumar N, Ahuja A, Jain A, Ray R, Sarkar C and Gupta SD: Primary malignant melanoma at unusual sites: An institutional experience with review of literature. Melanoma Res 20: 233-239, 2010.

12 Nobbenhuis MA, Lalondrelle S, Larkin $\mathrm{J}$ and Banerjee S: Management of melanomas of the gynaecological tract. Curr Opin Oncol 26: 508-513, 2014.

13 Hao C, Tian J, Liu H, Li F, Niu H and Zhu B: Efficacy and safety of anti-PD-1 and anti-PD-1 combined with anti-CTLA-4 immunotherapy to advanced melanoma: A systematic review and meta-analysis of randomized controlled trials. Medicine 96(26): e7325, 2017.

14 Skovsted S, Nielsen K and Blaakær J: Melanomas of the vulva and vagina. Dan Med J 64(3): A5336, 2017.

15 Kalampokas E, Kalampokas $\mathrm{T}$ and Damaskos C: Primary vaginal melanoma, a rare and aggressive entity. A case report and review of the literature. In Vivo 31(1): 133-139, 2017.

16 Agarwal $\mathrm{P}$ and Kaushal M: Cytology of primary vaginal melanoma: An unusual report on fine-needle aspiration. Diagn Cytpathol 45(3): 252-256, 2017.

17 Lee JH, Yun J, Seo JW, Bae GE, Lee JW and Kim SW: Primary malignant melanoma of cervix and vagina. Obstet Gynecol Sci 59(5): 415-420, 2016.

18 Pankaj S, Kumari A, Nazneen S, Choudhary V and Kumari S: Malignant melanoma of vagina: a report and review of literature. J Obstet Gynaecol India 66(5): 394-396, 2016.

19 Rema P, Suchetha S and Ahmed I: Primary malignant melanoma of vagina treated by total pelvic exenteration. Indian J Surg 78(1): 65, 2016.

20 Todo Y, Okamoto K, Suzuki Y, Minobe S and Kato H: Radicality of initial surgery for primary malignant melanoma of the vagina. Melanoma Res 26(2): 173-180, 2016.

21 Stefanovic A, Jeremić J, Jeremić K, Likić I, Mitrović M and Stojnić J: Primary melanoma of the vagina: a case report and review of literature. Eur J Gynaecol Oncol 36(6): 755-757, 2015.

22 Ishida H, Nagai T, Sato S, Honda M, Uotani T, Samejima K, Hanaoka T, Akahori T, Takai Y and Seki H: Concomitant sentinel lymph node biopsy leading to abbreviated systematic lymphadenectomy in a patient with primary malignant melanoma of the vagina. Springerplus 4: 102, 2015.

23 Liu QY, Zeng YP, Lin XF, Liu ZF, Wu XF and Li HG: MRI findings in primary vaginal melanoma-a report of four cases. Clin Imaging 39(3): 533-537, 2015.
24 Seifried S, Haydu LE, Quinn MJ, Scolyer RA, Stretch JR and Thompson JF: Melanoma of the vulva and vagina: principles of staging and their relevance to management based on a clinicopatho-logic analysis of 85 cases. Ann Surg Oncol 22(6): 1959-1966, 2015.

25 Tsvetkov Ch, Gorchev G, Tomov S, Hinkova N, Nikolova M and Veselinova T: Primary malignant melanoma of the vagina and treatment options: a case report. Akush Ginekol 53(7): 35-40, 2014.

26 Chen L, Xiong Y, Wang H, Liang L, Shang H and Yan X: Malignant melanoma of the vagina: A case report and review of the literature. Oncol Lett 8(4): 1585-1588, 2014.

27 Karasawa K, Wakatsuki M, Kato S, Kiyohara H, Kamada T and Working Group for Gynecological Tumors: Clinical trial of carbon ion radiotherapy for gynecological melanoma. J Radiat Res 55(2): 343-350, 2014.

28 Xia L, Han D, Yang W, Li J, Chuang L and Wu X: Primary malignant melanoma of the vagina: a retrospective clinico-pathologic study of 44 cases. Int J Gynecol Cancer 24(1): 149-155, 2014.

29 Chaudhuri S, Das D, Chowdhury S and Gupta AD: Primary malignant melanoma of the vagina: A case report and review of literature. South Asian J Cancer 2(1): 4, 2013.

30 Huang Q, Huang H, Wan T, Deng T and Liu J: Clinical outcome of 31 patients with primary malignant melanoma of the vagina. J Gynecol Oncol 24(4): 330-335, 2013.

31 Kirschner AN, Kidd EA, Dewees T and Perkins SM: Treatment approach and outcomes of vaginal melanoma. Int J Gynecol Cancer 23(8): 1484-1489, 2013.

$32 \mathrm{Kahr}$ HS, Mejlgaard E and Lund B: Primary malignant melanoma of the vagina. Ugeskr Laeger 175(3): 133-134, 2013.

33 Chauhan N, Gaur DS and Pathak VP: round cell vaginal malignant melanoma: A rare entity. Sultan Qaboos Univ Med J 12(4): 508-511, 2012.

34 Gauthier T, Uzan C, Gouy S, Kane A, Calvacanti A, Mateus C, Robert C, Kolb F and Morice P: Malignant melanoma of the vagina: pejorative location. Gynecol Obstet Fertil 40(5): 273-278, 2012.

35 Tcheung WJ, Selim MA, Herndon JE 2nd, Abernethy AP and Nelson KC: Clinicopathologic study of 85 cases of melanoma of the female genitalia. J Am Acad Dermatol 67(4): 598-605, 2012.

36 Lin LT, Liu CB, Chen SN, Chiang AJ, Liou WS and Yu KJ: Primary malignant melanoma of the vagina with repeated local recurrences and brain metastasis. J Chin Med Assoc 74(8): 376379, 2012.

37 Albareda J, Olier C and Alemany I: Primary melanoma of the vagina. A clinical case. J Turk Germ Gynecol Assoc 12(1): 5052, 2011.

38 Frumovitz M, Etchepareborda M, Sun CC, Soliman PT, Eifel PJ, Levenback CF and Ramirez PT: Primary malignant melanoma of the vagina. Obstet Gynecol 116(6): 1358-1365, 2010.

39 Greggi S, Losito S, Pisano C, Desicato S and Scaffa C: Malignant melanoma of the vagina: report of two cases and review of the literature. Int Surg 95(2): 120-125, 2010.

40 Samolis S, Panagopoulos P, Kanellopoulos N, Papastefanou I, Karadaglis S and Katsoulis M: Primary melanoma of the vagina: a case report. Eur J Gynaecol Oncol 31(2): 233-234, 2010.

41 Biswas B, Garain P, Dasgupta S, Sen S, Biswas P and Dasgupta C: Malignant melanoma of vagina-a case report. J Indian Med Assoc 107(4): 247-248, 2009.

42 Gungor T, Altinkaya SO, Ozat M, Bayramoglu $\mathrm{H}$ and Mollamahmutoglu L: Primary malignant melanoma of the female genital tract. Taiwan J Obstet Gynecol 48(2): 169-175, 2009. 
43 Baloglu A, Bezircioglu I, Cetinkaya B and Yavuzcan A: Primary malignant melanoma of the vagina. Arch Gynecol Obstet 280(5): 819-822, 2009.

44 Luo LM, Huang HF, Pan LY, Shen K, Wu M and Xu L: Clinical analysis of 42 cases of primary malignant tumor in vagina. Zonghua Fu Chan Ke Za Zhi 43(12): 923-927, 2008.

45 Takai N, Kai N, Hirata Y, Kashima K and Narahara H: Primary malignant melanoma of the vagina. Eur J Gynaecol Oncol 29(5): 558-559, 2008.

46 Mukhopadhyay S, Ghosh S, Siddhartha D and Mitra PK: A clinicopathological study of malignant melanoma with special reference to atypical presentation. Indian J Pathol Microbiol 51(4): 485-488, 2008.

47 Grenader T, Isacson R, Reinus C, Rosengarten O, Barenholz O, Hyman J, Gabizon A and Beller U: Primary amelanotic melanoma of the vagina. Onkologie 31(8-9): 474-476, 2008.

48 Schmidt M, Honig A, Schwab M, Adam P and Dietl J: Primary vaginal melanoma: a case report and literature review. Eur J Gynaecol Oncol 29(3): 285-288, 2008.

49 Ghosh A, Pradhan S, Swami R, Kc SR and Talwar O: Primary malignant melanoma of vagina - a case report with review of literature. J Nepal Med Assoc 46(168): 203-205, 2007.

50 An JS, Wu LY, Li N, Li B, Yu GZ and Liu LY: Clinical analysis of 42 cases of primary malignant melanoma in female genital tract. Zonghua Fu Chan Ke Za Zhi 42(5): 320-324, 2007.

51 Fulciniti F, Ascierto PA, Simeone E, Bove P, Losito S, Russo S, Gallo MS and Greggi S: Nevoid melanoma of the vagina: report of one case diagnosed on thin layer cytological preparations. Cytojournal 4: 14, 2007.

52 Ohno T, Kato S, Sasaki E, Mizutani K and Tsujii H: Carbon ion radiotherapy for vaginal malignant melanoma: a case report. Int J Gynecol Cancer 17(5): 1163-1166, 2007.

53 Betschart C, von Orelli S, Mihic D and Fink D: Primary malignant melanoma of the vagina-case report and review of the literature. Gynakol Geburtshilfliche Rundsch 47(1): 39-44, 2007.

54 Androutsopoulos G, Adonakis G, Ravazoula P and Kourounis G: Primary malignant melanoma of the vagina: a case report. Eur $\mathrm{J}$ Gynaecol Oncol 26(6): 661-662, 2005.

55 Gökaslan H, Sişmanoğlu A, Pekin T, Kaya H and Ceyhan N: Primary malignant melanoma of the vagina: a case report and review of the current treatment options. Eur J Obstet Gynecol Reprod Biol 121(2): 243-248, 2005.

56 Oguri H, Izumiya C, Maeda N, Fukaya T and Moriki T: A primary amelanotic melanoma of the vagina, diagnosed by immunohistochemical staining with HMB-45, which recurred as a pigmented melanoma. J Clin Pathol 57(9): 986-988, 2004.

57 Moros ML, Ferrer FP, Mitchell MJ, Romeo JA and Lacruz RL: Primary malignant melanoma of the vagina. Poor response to radical surgery and adjuvant therapy. Eur J Obstet Gynecol Reprod Biol 113(2): 248-250, 2004.

58 Siu SS, Lo KW, Chan AB, Yu MY and Cheung TH: Nodal detection in malignant melanoma of the vagina using laparoscopic ultrasonography. Gynecol Oncol 92(3): 985-988, 2004.

59 Mishra M, Chawla SC, Chawla S, Basu SM and Tata R: Malignant melanoma of the vagina: report of two cases. Indian J Pathol Microbiol 46(1): 71-73, 2003.

60 Kim H, Jung SE, Lee EH, Kang SW: Case report: magnetic resonance imaging of vaginal malignant melanoma. J Comput Assist Tomogr 27(3): 357-360, 2003.
61 Gupta D, Malpica A, Deavers MT and Silva EG: Vaginal melanoma: a clinicopathologic and immunohistochemical study of 26 cases. Am J Surg Pathol 26(11): 1450-1457, 2002.

62 Nakagawa S, Koga K, Kugu K, Tsutsumi O and Taketani Y: The evaluation of the sentinel node successfully conducted in a case of malignant melanoma of the vagina. Gynecol Oncol 86(3): 387-389, 2002.

63 Piura B, Rabinovich A and Yanai-Inbar I: Primary malignant melanoma of the vagina: case report and review of literature. Eur J Gynaecol Oncol 23(3): 195-198, 2002.

64 Panek G, Bidziński M and Nasierowska-Guttmeier A: Primary vaginal and uterine melanoma-a case of long term survival after local excision and vaginal brachytherapy. Ginekol Pol 72(12A): 1501-1506, 2001.

65 Fan SF, Gu WZ and Zhang JM: Case report: MR findings of malignant melanoma of the vagina. Br J Radiol 74(881): 445447, 2001.

66 Takehara M, Ito E, Saito T, Nishioka Y, Ashihara K, Yamashita S, Koizumi M and Kudo R: HMB-45 staining for cytology of primary melanoma of the vagina. A case report. Acta Cytol 44(6): 1077-1080, 2000.

67 Cobellis L, Calabrese E, Stefanon B and Raspagliesi F: Malignant melanoma of the vagina. A report of 15 cases. Eur $\mathbf{J}$ Gynaecol Oncol 21(3): 295-297, 2000.

$68 \mathrm{Li} \mathrm{Y,} \mathrm{Li} \mathrm{M}$ and Wu Q: Clinical analysis of 25 cases of primary vaginal malignant melanoma. Zhonghua Fu Chan Ke Za Zhi 34(3): 162-164, 1999.

69 Rodier JF, Janser JC, David E, Routiot T and Ott G: Radiopharmaceutical-guided surgery in primary malignant melanoma of the vagina. Gynecol Oncol 75(2): 308-309, 1999.

70 Irvin WP Jr., Bliss SA, Rice LW, Taylor PT Jr and Andersen WA: Malignant melanoma of the vagina and locoregional control: radical surgery revisited. Gynecol Oncol 71(3): 476-480, 1998.

71 Creasman WT, Phillips JL and Menck HR: The National Cancer Data Base report on cancer of the vagina. Cancer 83(5): 10331040, 1998.

72 Petru E, Nagele F, Czerwenka K, Graf AH, Lax S, Bauer M, Pehamberger $\mathrm{H}$ and Vavra $\mathrm{N}$ : Primary malignant melanoma of the vagina: long-term remission following radiation therapy. Gynecol Oncol 70(1): 23-26, 1998.

73 Stellato G, Iodice F, Casella G, Fortuna G, Tramontana R, di Bonito $\mathrm{M}$ and Tramontana S: Primary malignant melanoma of the vagina: case report. Eur J Gynaecol Oncol 19(2): 186-188, 1998.

74 Wang Y, Chang W and Pu D: Clinical analysis of 15 patients with primary malignant melanoma in the genital tract. Zhonghua Fu Chan Ke Za Zhi 32(4): 226-228, 1997.

75 Zarcone R, Bellini P, Carfora E and Cardone A: A case of malignant melanoma of the vagina during pregnancy: immunological problems. Eur J Gynaecol Oncol 18(2): 136-138, 1997.

76 Liu L, Li X and Hong W: Primary malignant melanoma of the vagina: a report of 22 cases. Zhonghua Fu Chan Ke Za Zhi 18(5): 385-387, 1996.

77 Robert C, Long G, Brady B, Dutriaux C, Maio M, Mortier L, Hassel J, Rutkowski P, McNeil C and Kalinka-Warzocha E: Nivolumab in previously untreated melanoma without mutation. N Engl J Med 372(4): 320-330, 2015.

Received September 10, 2017

Revised October 7, 2017 Accepted October 12, 2017 